\title{
3T MRI evaluation of regional catecholamine-producing tumor-induced myocardial injury
}

\author{
Satoshi Higuchi ${ }^{1}$, Hideki Ota ${ }^{1}$, Takuya Ueda ${ }^{1}$, Yuta Tezuka ${ }^{2}$, Kei Omata ${ }^{2}$, Yoshikiyo Ono ${ }^{2}$, Ryo Morimoto², \\ Masataka Kudo' ${ }^{2}$ Fumitoshi Satoh ${ }^{2,3}$ and Kei Takase ${ }^{1}$ \\ 'Department of Diagnostic Radiology, Tohoku University Hospital, Sendai, Miyagi, Japan \\ 2Division of Nephrology, Endocrinology and Vascular Medicine, Department of Medicine, Tohoku University Hospital, Sendai, Miyagi, Japan \\ ${ }^{3}$ Division of Clinical Hypertension, Endocrinology and Metabolism, Tohoku University Graduate School of Medicine, Sendai, Miyagi, Japan \\ Correspondence should be addressed to H Ota: h-ota@rad.med.tohoku.ac.jp
}

\begin{abstract}
Objective: Regional differences in cardiac magnetic resonance, which can reveal catecholamine-induced myocardial injury in patients with pheochromocytoma, have not yet been assessed using 3T magnetic resonance imaging. We evaluated these differences using myocardial T1-mapping and strain analysis.

Design and Methods: We retrospectively reviewed 16 patients newly diagnosed with catecholamine-producing tumors (CPT group) and 16 patients with essential hypertension (EH group), who underwent cardiac magnetic resonance imaging between May 2016 and March 2018. We acquired 3T magnetic resonance cine and native T1-mapping images and performed feature-tracking-based strain analysis in the former. Results: Global cardiac function, morphology, global strain and peak strain rate were similar, but end-diastolic wall thickness differed between groups (CPT vs EH: $10.5 \pm 1.7$ vs $12.6 \pm 2.8 \mathrm{~mm} ; P<0.05)$. Basal, but not apical, circumferential strain was significantly higher in the CPT than the EH group (19.4 \pm 3.2 vs $16.8 \pm 3.6 \% ; P<0.05)$. Native T1 values were significantly higher in CPT than in EH patients, in both the basal septum $(1307 \pm 48$ vs $1241 \pm 45 \mathrm{~ms} ; P<0.01)$ and the apical septum $(1377 \pm 59$ vs $1265 \pm 58 \mathrm{~ms} ; P<0.01)$ mid-walls. In the CPT, but not in the EH group, native T1 values in the apical wall were significantly higher than those in the basal wall $(P<0.01)$. Conclusion: 3 T magnetic resonance-based T1-mapping can sensitively detect subclinical catecholamine-induced myocardial injury; the influence of catecholamines may be greater in the apical than in the basal wall.
\end{abstract}

\section{Key Words}

- catecholamine-induced myocardial injury

- myocardial strain

- myocardial T1-mapping

- regional difference

\section{Introduction}

Pheochromocytomas and paragangliomas are catecholamine-producing tumors (CPTs). The prevalence of pheochromocytoma in patients with hypertension in general outpatient clinics varies between 0.2 and $0.6 \%$ (1). Excess catecholamines are secreted from the adrenal gland or extra-adrenal chromaffin cells in CPTs, spontaneously. Patients with these tumors may present with various clinical symptoms, such as episodic headaches, sweating, tachycardia, palpitations and paroxysmal hypertension (2). The increase in catecholamine production can also lead to high morbidity and mortality, due to catastrophic cardiovascular complications, including myocardial ischemia, aortic dissection, stroke, hypertensive crisis and peripheral ischemia $(3,4,5,6)$.

The excess catecholamines and their metabolites have direct toxic as well as receptor-mediated effects on

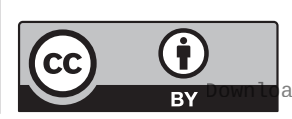

This work is licensed under a Creative Commons Attribution 4.0 International License. 
myocardial tissue $(7,8)$. Progressive cardiac injury leads to cardiac remodeling and decreased cardiac function, independent of the hypertensive effect, and results in catecholamine-induced cardiomyopathy (9). The prevalence of catecholamine-induced cardiomyopathy in patients with pheochromocytoma is $10-11 \%(10,11)$. Patients with catecholamine-induced cardiomyopathy often have a poor prognosis due to fatal arrhythmias, heart failure and circulatory collapse $(11,12)$. However, patients without cardiac remodeling and dysfunction can also have catecholamine-induced cardiac damage. Previous reports have indicated that the sudden release of catecholamines from the pheochromocytoma is associated with QT prolongation and ventricular tachycardia (VT); torsades de pointes or VT have been observed even in young pheochromocytoma patients without cardiac dysfunction $(13,14,15)$.

Cardiac magnetic resonance (CMR) imaging is a valuable tool for non-invasive assessment of cardiac morphology and function, and characterization of myocardial tissue. Cine magnetic resonance (MR) images allow evaluation of left ventricular volumetric parameters as well as wall motion velocity, myocardial strain and strain rate, which can facilitate detection of local or global dysfunction using wall motion tracking methods (16). In terms of tissue characterization, myocardial T1-mapping provides myocardial longitudinal (spin-lattice) relaxation times reflecting myocardial tissue properties, such as edema, fibrosis or fat infiltration, without the use of contrast medium. This technique is a sensitive tool for detecting local and diffuse myocardial pathophysiological changes (17).

A recent report has shown that patients with pheochromocytoma had a lower left ventricular ejection fraction, lower peak systolic circumferential strain, lower diastolic strain rate and higher myocardial native T1 values than healthy controls and patients with hypertension, based on the results obtained using a $1.5 \mathrm{~T}$ MR scanner (18). However, there have been no reports of 3T CMR imaging providing higher signal-to-noise ratio than 1.5T CMR imaging in CPT patients. The previous 1.5T MR study used myocardial tagging to obtain the radial and circumferential strains and did not show the longitudinal strain.

Furthermore, the left ventricle contains apicalbasal gradients of $\beta$ adrenoreceptors ( $\beta$ ARs) (which are responsible for increased responsiveness to epinephrine) and sympathetic innervation, with the apex characterized by the highest $\beta A R$ concentration (19). There is a hypothesis that the distribution of $\beta$ ARs contributes to abnormal systolic wall motion in Takotsubo cardiomyopathy (TC), where hypokinetic apical wall with ballooning and hyperkinetic basal wall are observed (20). A recent study reported that acute Takotsubo-like cardiomyopathy was found in up to $3 \%$ of patients with functional pheochromocytoma and paraganglioma (21), whereas only $0.02 \%$ of all hospitalized patients suffered from TC (22). However, the regional distribution of the degree of catecholamine-induced myocardial damage has not been evaluated in patients with CPTs.

We hypothesized that CMR may be able to identify the regional variance of the catecholamine-induced myocardial damage in patients with CPTs without contrast medium. We aimed to evaluate whether CMR could detect subclinical catecholamine-induced cardiac injury in patients with CPTs, as compared to patients with essential hypertension (EH), using a 3T MR scanner. We also evaluated whether there were differences in the degree of myocardial injury between the basal and apical regions using myocardial T1-mapping and wall motion tracking.

\section{Materials and methods}

\section{Ethics}

This retrospective study was approved by the Ethics Committee of Tohoku University School of Medicine (\#2018-1-133). The requirement for obtaining written informed consent from the patients was waived by the ethics committee.

\section{Patients}

Sixteen consecutive patients with newly diagnosed CPT, who underwent CMR examinations at our institution from March 2016 to May 2018 and who did not meet the exclusion criteria, were included in the study. The presence of CPT was diagnosed by endocrinologists based on serum and urinary biochemical tests, ${ }^{123}$ I metaiodobenzylguanidine scintigraphy and MR imaging. Another 16 consecutive patients with $\mathrm{EH}$, who underwent CMR imaging within the same period of time, were also included. These patients were referred to our institution because of refractory hypertension, but were diagnosed with $\mathrm{EH}$ by the same endocrinologists after ruling out secondary causes of hypertension. Exclusion criteria were a history of cardiac events (heart failure, myocardial infarction, valvular disease and arrhythmias) and severe renal dysfunction (estimated glomerular filtration rate $<30 \mathrm{~mL} / \mathrm{min})$.

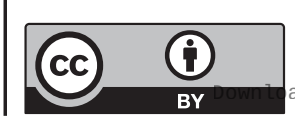

This work is licensed under a Creative Commons Attribution 4.0 International License. ded from Bioscientifica.com at $04 / 26 / 2023$ 07:41:46AM 
Demographic characteristics of the patients were collected; these included sex, age, BMI, body surface area, family history of $\mathrm{EH}$, medical history of diabetes mellitus, smoking history, number of antihypertensive drugs, systolic blood pressure (measured at home) and disease duration (the interval from the appearance of subjective symptoms or detection of hypertension to the diagnosis). In the CPT group, 24-h urinary metanephrine and normetanephrine levels were evaluated to determine the biochemical phenotype (adrenergic or noradrenergic). Patients are classified as adrenergic if the increment of metanephrine exceeded $5 \%$ of the combined metanephrine and normetanephrine increments. Patients in whom these criteria were not fulfilled and in whom normetanephrine levels exceeded the upper limits of normal were classified as noradrenergic (23).

\section{CMR protocol}

All patients were imaged with a 3T whole body MR scanner (MAGNETOM Trio A Tim System; Siemens Healthineers) before surgery. Scanning protocols included cine and precontrast T1 maps. Native T1 maps were acquired using a modified Look-Locker inversion recovery (MOLLI) technique. Cine images were acquired at 20 frames per slice per beat in short-axis, long-axis and four-chamber views. Details of the parameters in cine and T1-mapping are provided in Supplementary Table 1 (see section on supplementary data given at the end of this article). To measure the longer T1 time accurately, independent of patient heart rate for native T1 values, we applied the (8(2)2) scheme indicating two inversion pulses with acquisition of images for eight heart beats, followed by a recovery of two heart beats and a second inversion pulse with images acquired for two heart beats. In-plane motion correction was performed and T1 maps were automatically generated.

\section{Image analysis}

We analyzed MR images using a workstation (Ziostation2; Ziosoft, Tokyo, Japan). We measured the end-systolic and end-diastolic ventricular septal wall thickness; left ventricle ejection fraction (LVEF); the end-diastolic, endsystolic and stroke volume index (EDVI, ESVI and SI, respectively); and the cardiac index (CI) from short-axis cine images. The left ventricular myocardial mass index (LVMI) was also acquired from short-axis cine images, using Simpson's technique. We analyzed myocardial strain and strain rate using the MR Wall Motion Tracking application (Vitrea, Canon Medical Systems, Otawara, Japan). Radial strain and circumferential strain were derived from the short-axis images and longitudinal strain was derived from the two- and four-chamber images. We used the average of the longitudinal strain from the two- and four-chamber images as the global longitudinal strain. We also evaluated basal and apical wall strain from segmented strain data.

Two radiologists ( $\mathrm{H} \mathrm{O}$ and $\mathrm{S} \mathrm{H}$, with 16 and 5 years of experience, respectively) independently measured native $\mathrm{T} 1$ values, for analysis of interobserver reproducibility. Regions of interest for evaluation of native $\mathrm{T} 1$ values were placed on the mid-wall of the basal septum and the mid-wall of the apical septum, using the short-axis and four-chamber T1 images (Fig. 1). T1 values measured by a single reviewer were considered to be representative of individual segments. Reviewers were blinded to the patients' demographics.

\section{Statistical analysis}

Descriptive statistics are presented as means and standard deviations (s.D.) for continuous variables and as the number of cases and percentages per group for categorical variables. Nonparametric data are shown as medians
A

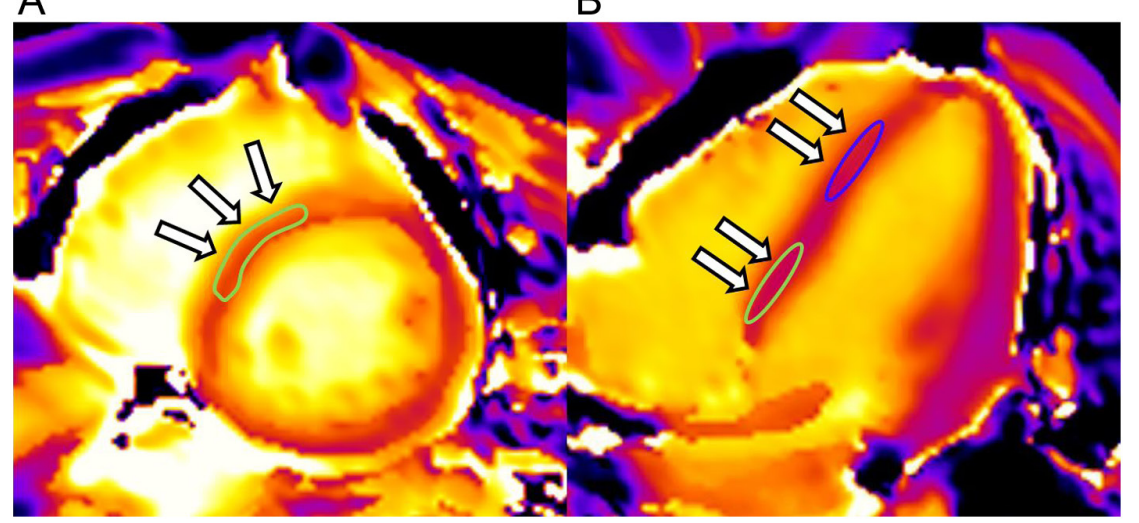

Figure 1

Images from a 49-year-old male with pheochromocytoma. Sample of region of interest for evaluation of native T1 values in the basal septum (A and B) and apical septum (B). Arrows indicate the region of interest for native T1 measurement. https://ec.bioscientifica.com https://doi.org/10.1530/EC-18-0553 (c) 2019 The authors Published by Bioscientifica Ltd

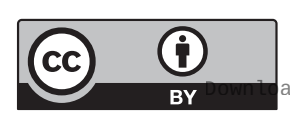

This work is licensed under a Creative Commons Attribution 4.0 International License. ded from Bioscientifica.com at 04/26/2023 07:41:46AM 
with interquartile ranges (IQRs). The normality of MR parameters and patient demographic data were tested using the Kolmogorov-Smirnov test. Variables between $\mathrm{CPT}$ and EH groups were compared using Student's $t$-test or the Mann-Whitney $U$ test. For dichotomous data, Fisher's exact test was used to analyze the difference of proportions between the groups. We analyzed paired data using the paired $t$-test or the Wilcoxon signed-rank test. Interobserver reproducibility of native $\mathrm{T} 1$ values was evaluated using intraclass correlation coefficients (ICCs). Based on the 95\% confident intervals (CI) of the ICCs, values less than 0.5 , values $0.5-0.75,0.75-0.9$ and those greater than 0.9 were considered indicative of poor, moderate, good and excellent reliability, respectively. Statistical analyses were performed using SPSS version 21.0 (IBM). $P<0.05$ was considered statistically significant.

\section{Results}

\section{Patient characteristics}

Among the included 16 patients with CPT, 15 underwent surgical resection; histopathological examinations revealed pheochromocytoma in 13 patients, paraganglioma in 1 patient and ganglioneuroblastoma in 1 patient. The other patient had a mediastinal tumor that was clinically diagnosed as a paraganglioma after positive biochemical tests and scintigraphy findings; this patient did not undergo surgery, as the patient experienced aortic dissection during the presurgical period. The baseline patient characteristics are summarized in Table 1.

Table 1 Patient demographic characteristics.

\begin{tabular}{|c|c|c|c|}
\hline & $\begin{array}{l}\text { CPT group } \\
(n=16)\end{array}$ & $\begin{array}{l}\text { EH group } \\
(n=16)\end{array}$ & $P$ value \\
\hline Age (years) & $52 \pm 13$ & $53 \pm 14$ & 0.87 \\
\hline Female $(\%(n))$ & $62(10 / 16)$ & $50(8 / 16)$ & 0.72 \\
\hline $\mathrm{BMI}\left(\mathrm{kg} / \mathrm{m}^{2}\right)^{*}$ & $22.6 \pm 3.2$ & $25.8 \pm 3.8$ & 0.02 \\
\hline $\mathrm{BSA}\left(\mathrm{m}^{2}\right)$ & $1.6(1.6-1.7)$ & $1.7(1.5-1.8)$ & 0.62 \\
\hline $\begin{array}{l}\text { Family history of } \\
\text { hypertension }(\%(n))\end{array}$ & $75(12 / 16)$ & $88(14 / 16)$ & 0.65 \\
\hline $\mathrm{DM}(\%(n))$ & $19(3 / 16)$ & $13(2 / 16)$ & 1.00 \\
\hline $\begin{array}{l}\text { Smoking history } \\
(\%(n))\end{array}$ & $50(8 / 16)$ & $69(11 / 16)$ & 0.47 \\
\hline $\begin{array}{l}\text { Number of } \\
\text { antihypertensive } \\
\text { drugs (n) }\end{array}$ & $2(0-2)$ & $2(1-2)$ & 0.64 \\
\hline Home sBP (mmHg) & $130(116-150)$ & $130(120-148)$ & 0.90 \\
\hline Disease duration $(\mathrm{y})^{*}$ & $2.3(1-5.5)$ & $6.5(2.3-16.8)$ & 0.02 \\
\hline
\end{tabular}

Table 2 Basic cardiac function and morphology.

\begin{tabular}{|c|c|c|c|}
\hline & CPT group & EH group & $P$ value \\
\hline LV EDWT $(\mathrm{mm})^{*}$ & $10.5 \pm 1.6$ & $12.4 \pm 2.8$ & 0.03 \\
\hline LV ESWT $(\mathrm{mm})$ & $13.8 \pm 2.8$ & $15.7 \pm 2.5$ & 0.053 \\
\hline LV EF (\%) & $58.3 \pm 7.6$ & $57.6 \pm 7.9$ & 0.82 \\
\hline LV EDVI $\left(\mathrm{mL} / \mathrm{m}^{2}\right)$ & $77.0 \pm 9.0$ & $69.8 \pm 15.3$ & 0.11 \\
\hline LV ESVI $\left(\mathrm{mL} / \mathrm{m}^{2}\right)$ & $33.7(24.3-38.1)$ & $34.6(24.8-36.5)$ & 0.78 \\
\hline LV SI (mL/m²) & $44.9 \pm 6.6$ & $41.2 \pm 7.7$ & 0.16 \\
\hline $\mathrm{LV} \mathrm{Cl}\left(\mathrm{mL} / \mathrm{min} / \mathrm{m}^{2}\right)$ & 3109 (2776-3672) & 2753 (2573-3091) & 0.07 \\
\hline LV MI (g/m²) & $49.0 \pm 13.2$ & $57.9 \pm 17.7$ & 0.12 \\
\hline
\end{tabular}

$\mathrm{Cl}$, cardiac index; CPT, catecholamine-producing tumor; EDVI, enddiastolic volume index; EDWT, end-diastolic wall thickness; EF, ejection fraction; EH, essential hypertension; ESVI, end-systolic volume index; ESWT, end-systolic wall thickness; LV, left ventricle; MI, mass index; SI, systolic volume index. Bold indicates statistical significance.

None of the patients from the $\mathrm{EH}$ group were excluded from the analysis. The EH group demonstrated a significantly larger BMI $\left(25.3 \pm 3.8\right.$ vs $22.6 \pm 3.2 \mathrm{~kg} / \mathrm{m}^{2}$; $P=0.02$ ) and longer disease duration than the CPT group (6.5 (IQR, 2.3-16.8) vs 2.3 (IQR, 1-5.5) years; $P=0.02$ ). There were no significant differences between the two groups with regard to the other demographic parameters.

\section{Left ventricular morphology and function}

Left ventricular (LV) end-diastolic walls were significantly thicker in the $\mathrm{EH}$ group than in the CPT group $(12.6 \pm 2.8 \mathrm{~mm}$ vs $10.5 \pm 1.7 \mathrm{~mm} ; P=0.025)$. Other $\mathrm{LV}$ functional parameters (end-systolic wall thickness, LVEF, EDVI, ESVI, SI, CI, LVMI) demonstrated no significant differences between the two groups (Table 2).

\section{LV myocardial strain}

Global strains of the three directions and the systolic and diastolic peak strain rates demonstrated no significant differences between the two groups. In segmental strain analysis, the CPT group demonstrated significantly higher circumferential strain of the basal wall than the EH group $(19.4 \pm 3.2 \%$ vs $16.8 \pm 3.6 \% ; P=0.04)$; however, apical circumferential strain was not significantly different $(25.0 \pm 6 \%$ vs $22.6 \pm 5.6 \% ; P=0.25)$. There was also no significant difference in the radial and longitudinal strains in the apical and basal walls between the two groups (Table 3).

\section{Native T1 values}

The ICCs of the T1 values measured in all the patients were 0.93 (95\% CI: 0.85-0.96) in the basal segments and 0.99 (95\% CI: 0.989-0.997) in the apical segments,

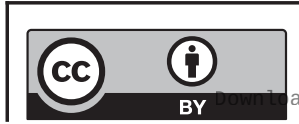

This work is licensed under a Creative Commons Attribution 4.0 International License. 
Table 3 Global and regional myocardial strain.

\begin{tabular}{|c|c|c|c|}
\hline & CPT group & EH group & $P$ value \\
\hline \multicolumn{4}{|l|}{ Global strain } \\
\hline \multicolumn{4}{|l|}{ Peak strain (\%) } \\
\hline Radial & $62.5 \pm 15.0$ & $64.0 \pm 26.8$ & 0.86 \\
\hline Circumferential & $-20.2 \pm 3.0$ & $-18.2 \pm 3.9$ & 0.11 \\
\hline Longitudinal & $-24.7 \pm 3.8$ & $-24.3 \pm 4.7$ & 0.78 \\
\hline \multicolumn{4}{|c|}{ Peak systolic strain rate $\left(\mathrm{s}^{-1}\right)$} \\
\hline Radial & $3.4 \pm 1.0$ & $3.7 \pm 1.7$ & 0.58 \\
\hline Circumferential & $-1.2 \pm 0.2$ & $-1.1 \pm 0.3$ & 0.29 \\
\hline Longitudinal & $-1.5 \pm 0.3$ & $-1.4 \pm 0.4$ & 0.67 \\
\hline \multicolumn{4}{|c|}{ Peak diastolic strain rate $\left(\mathrm{s}^{-1}\right)$} \\
\hline Radial & $-4.1 \pm 1.2$ & $-3.4 \pm 1.5$ & 0.21 \\
\hline Circumferential & $1.1 \pm 0.3$ & $1.0 \pm 0.4$ & 0.5 \\
\hline Longitudinal & $1.3(1.0-1.6)$ & $1.1(1.0-1.8)$ & 0.56 \\
\hline \multicolumn{4}{|l|}{ Regional strain } \\
\hline \multicolumn{4}{|l|}{ Basal peak strain (\%) } \\
\hline Radial & $66.9 \pm$ & 65.7 & 0.82 \\
\hline Circumferential* & $-19.4 \pm 3.2$ & $-16.8 \pm 3.7$ & 0.04 \\
\hline Longitudinal & $-24.8 \pm 3.8$ & $-24.3 \pm 5.0$ & 0.76 \\
\hline \multicolumn{4}{|l|}{ Apical peak strain (\%) } \\
\hline Radial & $55.8 \pm 20.2$ & $68.4 \pm 31.7$ & 0.19 \\
\hline Circumferential & $-25.0 \pm 6.1$ & $-22.6 \pm 5.6$ & 0.25 \\
\hline Longitudinal & $-24.5 \pm 4.0$ & $-24.3 \pm 4.6$ & 0.91 \\
\hline
\end{tabular}

CPT, catecholamine-producing tumor; EH, essential hypertension. Bold indicates statistical significance.

indicating excellent reliability. Patients with CPT demonstrated significantly higher native $\mathrm{T} 1$ values than those with $\mathrm{EH}$, in the mid-wall of both the basal septum $(1307 \pm 48 \mathrm{~ms}$ vs $1241 \pm 45 \mathrm{~ms} ; P<0.01)$ and the apical septum $(1377 \pm 59 \mathrm{~ms}$ vs $1265 \pm 58 \mathrm{~ms} ; P<0.01)$. In the CPT group, native T1 values on the mid-wall of the apical septum were significantly higher than those on the basal septum $(1377 \pm 59 \mathrm{~ms}$ vs $1308 \pm 49 \mathrm{~ms} ; P<0.01)$; however, the EH group did not demonstrate significant differences in native $\mathrm{T} 1$ values between the apical and basal walls ( $1265 \pm 58 \mathrm{~ms}$ vs $1258 \pm 48 \mathrm{~ms} ; P=0.34)$ (Fig. 2 ). The differences in native T1 values in the apical and basal interventricular septum were significantly higher in the CPT group than in the EH group $(69 \pm 47 \mathrm{~ms}$ vs $10 \pm 29 \mathrm{~ms}$; $P<0.01)$ (Fig. 3).

\section{Correlation between MR parameters and biochemical phenotype}

Among 16 CPT patients, eight were the adrenergic type and eight were the noradrenergic type. The MR parameters were not significantly different between the two groups.

\section{Discussion}

In this 3T CMR study of patients with CPT, we showed that the native T1 values in patients with CPT were

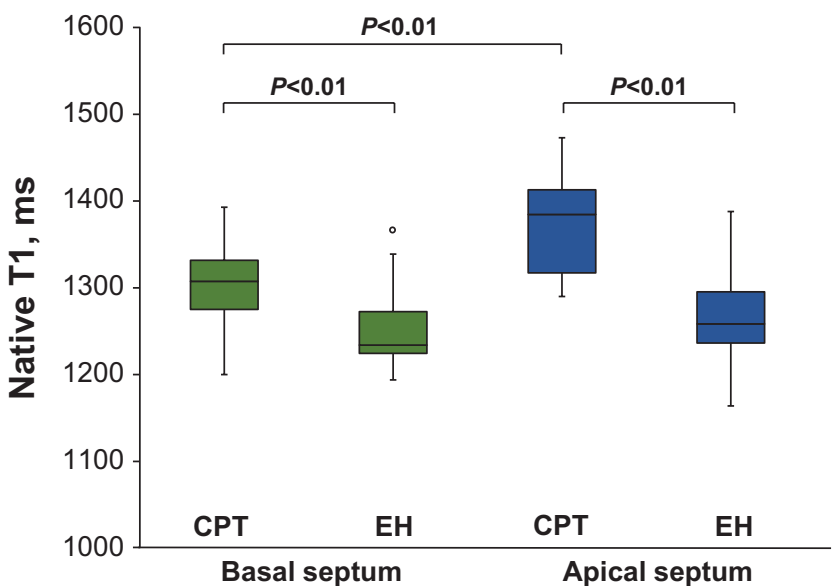

\section{Figure 2}

Box plots showing native T1 values in the basal and apical septal walls of the CPT and EH groups. Box plots show the medians, quartiles, ranges and outliers. Comparison between the CPT and EH groups was performed using Student's $t$-test, and between the basal and apical septum in the same group using the paired $t$-test. Native T1 values in the CPT group were significantly higher than those in the EH group in both the basal septum and apical septum. In the CPT group, native T1 values in the apical septum appeared significantly higher than those in the basal septum; however, there were no significant differences in the values between the apical septum and basal septum in the EH group. * indicates statistical significance.

significantly higher than those in patients with EH. Additionally, the apical septum's native T1 values were significantly higher than those of the basal septum in the CPT group, whereas in the EH group, there were no significant differences between the apical and basal septum's native T1 values. Furthermore, patients

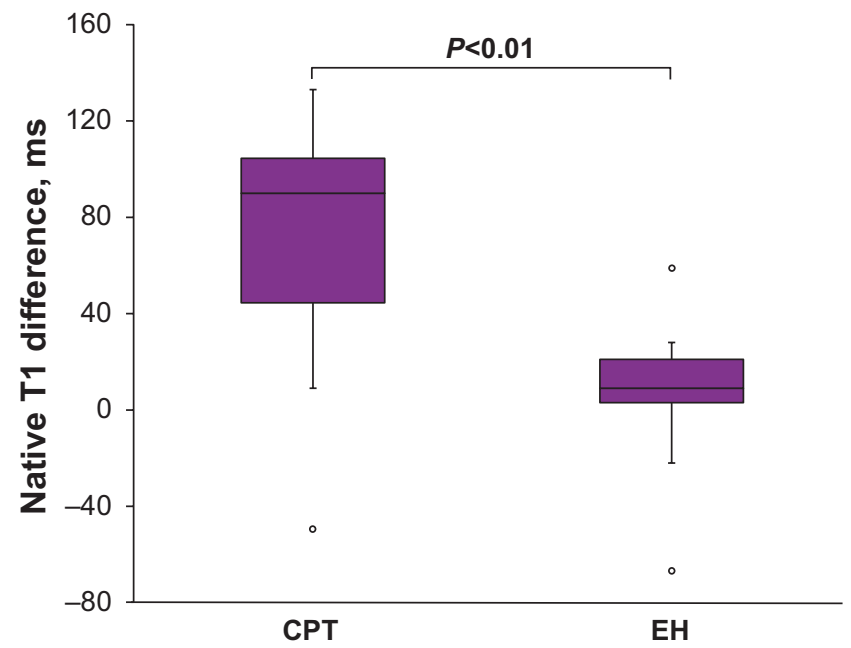

Figure 3

Box plots showing the difference in native T1 values between the apical septum and basal septum (basal values subtracted from apical values) in the CPT and EH groups. The difference in the CPT group was significantly greater than that in the EH group. * indicates statistical significance.

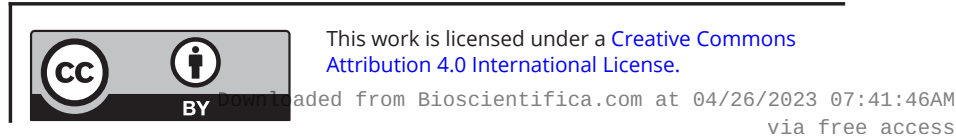


with CPT demonstrated significantly higher basal circumferential strain than those with $\mathrm{EH}$, despite comparable LV global function.

Ferreira et al. have reported a systemic study of catecholamine-induced cardiac injury using a $1.5 \mathrm{~T}$ MR scanner (18). They showed that patients with pheochromocytoma presented with lower LVEF, peak systolic circumferential strain and diastolic circumferential strain rate and higher native $\mathrm{T} 1$ values than $\mathrm{EH}$ patients. Our results were in line with the findings of Ferreira et al. in terms of the higher myocardial native T1 values in the CPT group than in the EH group. Another report indicated elevated native T1 values in severely hypertensive patients with LV hypertrophy compared to those in normotensive control subjects (24). However, in our study, there were no significant differences in the basic cardiac function between the two groups. The discrepancy between the studies may be due to the different study populations; our study may have enrolled patients with less severe cardiac damage than the study by Ferreira et al. Nonetheless, taken together, the results of the previous and present study indicate that T1-mapping can be a more sensitive tool than wall motion assessment for detecting catecholamineinduced cardiac injury.

Pathologically, catecholamines have been shown to cause myocardial injury, such as contraction band necrosis, via alpha-receptor-mediated vasoconstriction and oxygen-derived free radical injury, leading to myocyte membrane damage and death $(25,26)$. Moreover, autopsies of patients who died due to pheochromocytoma showed cardiac lesions, such as focal degeneration and necrosis of myocardial fibers, foci of inflammatory cells and diffuse myocardial edema, which were designated as catecholamine-induced myocarditis (26). The elevation of the native $\mathrm{T} 1$ values in our study may reflect the early stages of myocardial damage, with preserved cardiac function, in patients with CPT.

Although our study demonstrated no significant differences in global cardiac function or morphology between the CPT and EH groups, except for end-diastolic wall thickness, the LVEF of the patients in both groups were toward the lower limit of normal values as compared with the reported normal values obtained with CMR (27). Our subjects might have had a similar extent of mildly impaired LVEF, without a significant difference between the groups.

In terms of global strain analysis, the two groups in our study demonstrated no significant differences, but both groups might also have had impaired myocardial global circumferential strain as compared with the reported normal values for myocardial strain $(28,29)$. Previous studies have reported difficulty in matching hypertensive control subjects, because catecholamine-induced hypertension is highly variable and often paroxysmal, occurring on a background of sustained hypertension or presenting as normotension in between periods of hypertension $(6,30)$. Our patients in the control EH group had been taking more than two types of antihypertensive drugs for a significantly longer time than those in the CPT group and were referred for the work-up of hypertension; therefore, there might have been a selection bias in the control group, because mildly hypertensive patients might not have been referred to our endocrinology specialists. Previous reports have demonstrated a relationship of increased wall thickness with impaired myocardial strain $(31,32)$; our circumferential strain results in the EH group may be associated with a thickened myocardial wall.

Previous reports have shown that plasma catecholamine levels are markedly higher among patients with Takotsubo (stress-induced) cardiomyopathy (33), and mammalian hearts have a higher concentration of $\beta$-adrenoceptors in the apical myocardium, with the concentration decreasing in a gradient from the apex to the base $(19,34)$. These findings could explain the regional differences in response to high catecholamine levels, with circulating epinephrine having a greater influence on apical, relative to basal, function (20). The regional distribution of $\beta$-adrenoceptors might have also contributed to the significantly higher native $\mathrm{T} 1$ values in the apical wall and the relatively preserved circumferential strain in the basal wall in the CPT group. Our results may indicate that the apical wall may be prone to catecholamine-induced injury.

A previous study showed that native $\mathrm{T} 1$ values in patients with pheochromocytoma decreased after surgical resection along with $\mathrm{LV}$ mass regression, but did not completely normalize (18). Follow-up MR studies are needed to reveal whether resection of tumors improves cardiac function, elevated native $\mathrm{T} 1$ values and the difference in strain and native $\mathrm{T} 1$ values between the apical and basal septum. Moreover, clinical follow-up is also needed to determine the long-term clinical significance of these results.

This study had several limitations. First, although we demonstrated regional variance of T1 values in CPT patients, the sample size was relatively small. A larger study is warranted to confirm our results. Second, native $\mathrm{T} 1$ values are increased by several mechanisms, such as cardiomyocyte damage, enlarged extracellular space, intramyocardial edema and fibrosis (17).
This work is licensed under a Creative Commons Attribution 4.0 International License. ded from Bioscientifica.com at $04 / 26 / 2023$ 07:41:46AM 
We did not acquire late gadolinium enhancement (LGE) images and post-contrast T1-mapping to evaluate the extracellular volume fraction. A previous systemic MR study in pheochromocytoma patients showed that 58\% of patients presented with disseminated focal myocardial lesion pathologically, and $59 \%$ of these patients had a nonischemic pattern on LGE $(18,26)$. Therefore, we might have missed focal myocardial lesions, such as subclinical myocardial infarction. Such focal lesions might have contributed to the elevated native T1 values in the apical region. Nonetheless, native T1 values might comprehensively reflect several mechanisms underlying catecholamine-induced cardiac injury. Finally, endomyocardial biopsy was not performed in these patients, and thus direct histopathological correlation with the imaging findings was not possible.

In conclusion, T1-mapping using a 3T MR scanner is a sensitive tool for detecting subclinical catecholamineinduced cardiac injury and the influence of catecholamines on myocardial tissue, such as myocardial edema or fibrosis. We demonstrated that the injury may be greater in the apical than in the basal septum. Larger sample sizes and follow-up studies are needed to determine whether these subclinical findings are related to clinical prognosis and whether early intervention can minimize the catecholamine-induced myocardial injury.

\section{Supplementary data}

This is linked to the online version of the paper at https://doi.org/10.1530/ EC-18-0553.

\section{Declaration of interest}

The authors declare that there is no conflict of interest that could be perceived as prejudicing the impartiality of the research reported.

\section{Funding}

This research did not receive any specific grant from any funding agency in the public, commercial or not-for-profit sector.

\section{References}

1 Plouin PF, Amar L, Dekkers OM, Fassnacht M, Gimenez-Roqueplo AP, Lenders JW, Lussey-Lepoutre C, Steichen O \& Guideline Working Group. European Society of Endocrinology Clinical Practice Guideline for long-term follow-up of patients operated on for a phaeochromocytoma or a paraganglioma. European Journal of Endocrinology 2016174 G1-G10. (https://doi.org/10.1530/EJE-160033)

2 Bravo EL. Pheochromocytoma: new concepts and future trends. Kidney International 199140 544-556. (https://doi.org/10.1038/ ki.1991.244)
3 Gu YW, Poste J, Kunal M, Schwarcz M \& Weiss I. Cardiovascular manifestations of pheochromocytoma. Cardiology in Review 201725 215-222. (https://doi.org/10.1097/CRD.0000000000000141)

$4 \mathrm{Yu}$ R, Nissen NN \& Bannykh SI. Cardiac complications as initial manifestation of pheochromocytoma: frequency, outcome, and predictors. Endocrine Practice 201218 483-492. (https://doi. org/10.4158/EP11327.OR)

5 Zelinka T, Petrák O, Turková H, Holaj R, Strauch B, Kršek M, Vránková AB, Musil Z, Dušková J, Kubinyi J, et al. High incidence of cardiovascular complications in pheochromocytoma. Hormone and Metabolic Research 201244 379-384. (https://doi. org/10.1055/s-0032-1306294)

6 Galetta F, Franzoni F, Bernini G, Poupak F, Carpi A, Cini G, Tocchini L, Antonelli A \& Santoro G. Cardiovascular complications in patients with pheochromocytoma: A mini-review. Biomedicine and Pharmacotherapy 201064 505-509. (https://doi.org/10.1016/j. biopha.2009.09.014)

7 Varghese RT, John AM \& Paul TV. Catecholamine induced cardiomyopathy in pheochromocytoma. Indian Journal of Endocrinology and Metabolism 201317 733-735. (https://doi. org/10.4103/2230-8210.113771)

8 Kassim TA, Clarke DD, Mai VQ, Clyde PW \& Mohamed Shakir KM. Catecholamine-induced cardiomyopathy. Endocrine Practice 200814 1137-1149. (https://doi.org/10.4158/EP.14.9.1137)

9 Zhang R, Gupta D \& Albert SG. Pheochromocytoma as a reversible cause of cardiomyopathy: analysis and review of the literature. International Journal of Cardiology 2017249 319-323. (https://doi. org/10.1016/j.ijcard.2017.07.014)

10 Pappachan JM, Raskauskiene D, Sriraman R, Edavalath M \& Hanna FW. Diagnosis and management of pheochromocytoma: A practical guide to clinicians. Current Hypertension Reports 201416 442. (https://doi.org/10.1007/s11906-014-0442-z)

11 Park JH, Kim KS, Sul JY, Shin SK, Kim JH, Lee JH, Choi SW, Jeong JO \& Seong IW. Prevalence and patterns of left ventricular dysfunction in patients with pheochromocytoma. Journal of Cardiovascular Ultrasound 201119 76-82. (https://doi.org/10.4250/ jcu.2011.19.2.76)

12 Casey RT, Challis BG, Pitfield D, Mahroof RM, Jamieson N, Bhagra CJ, Vuylsteke A, Pettit SJ \& Chatterjee KC. Management of an acute catecholamine-induced cardiomyopathy and circulatory collapse: a multidisciplinary approach. Endocrinology, Diabetes and Metabolism Case Reports 20172017 17-0112. (https://doi. org/10.1530/EDM-17-0122)

13 Paulin FL, Klein GJ, Gula LJ, Skanes AC, Yee R \& Krahn AD. Qt prolongation and monomorphic vt caused by pheochromocytoma. Journal of Cardiovascular Electrophysiology 200920 931-934. (https:// doi.org/10.1111/j.1540-8167.2008.01405.x)

14 Zhang MM, Mao W, Wu D \& Liu P. Pheochromocytoma with ventricular tachycardia as the presenting symptom. Chinese Medical Journal 2016129 1505-1506. (https://doi.org/10.4103/03666999.183416)

15 Oruganti SS, Gambeer Rao M \& Pisapati VLNM. Adrenal and extra-adrenal pheochromocytomas presenting as life-threatening ventricular arrhythmias: report of three cases. Indian Heart Journal 201668 381-385. (https://doi.org/10.1016/j.ihj.2016.03.035)

16 Cheung YF. The role of 3D wall motion tracking in heart failure. Nature Reviews. Cardiology 20129 644-657. (https://doi.org/10.1038/ nrcardio.2012.128)

17 Moon JC, Messroghli DR, Kellman P, Piechnik SK, Robson MD, Ugander M, Gatehouse PD, Arai AE, Friedrich MG, Neubauer S, et al. Myocardial T1-mapping and extracellular volume quantification: a Society for Cardiovascular Magnetic Resonance (SCMR) and CMR Working Group of the European Society of Cardiology consensus statement. Journal of Cardiovascular Magnetic Resonance 20131592. (https://doi.org/10.1186/1532-429X-15-92) 
18 Ferreira VM, Marcelino M, Piechnik SK, Marini C, Karamitsos TD, Ntusi NAB, Francis JM, Robson MD, Arnold JR, Mihai R, et al. Pheochromocytoma is characterized by catecholamine-mediated myocarditis, focal and diffuse myocardial fibrosis, and myocardial dysfunction. Journal of the American College of Cardiology 201667 2364-2374. (https://doi.org/10.1016/j.jacc.2016.03.543)

19 Paur H, Wright PT, Sikkel MB, Tranter MH, Mansfield C, O'Gara P, Stuckey DJ, Nikolaev VO, Diakonov I, Pannell L, et al. High levels of circulating epinephrine trigger apical cardiodepression in a $\beta 2$-adrenoceptor/gi-dependent manner: a new model of takotsubo cardiomyopathy. Circulation 2012126 697-706. (https://doi. org/10.1161/CIRCULATIONAHA.112.111591)

20 Lyon AR, Rees PS, Prasad S, Poole-Wilson PA \& Harding SE. Stress (Takotsubo) cardiomyopathy - a novel pathophysiological hypothesis to explain catecholamine-induced acute myocardial stunning. Nature Clinical Practice Cardiovascular Medicine 20085 22-29. (https://doi.org/10.1038/ncpcardio1066)

21 Gagnon N, Mansour S, Bitton Y \& Bourdeau I. Takotsubolike cardiomyopathy in a large cohort of patients with pheochromocytoma and paraganglioma. Endocrine Practice 201723 1178-1192. (https://doi.org/10.4158/EP171930.OR)

22 Deshmukh A, Kumar G, Pant S, Rihal C, Murugiah K \& Mehta JL. Prevalence of takotsubo cardiomyopathy in the United States. American Heart Journal 2012164 66-71.e1. (https://doi.org/10.1016/j. ahj.2012.03.020)

23 Eisenhofer G, Pacak K, Huynh TT, Qin N, Bratslavsky G, Linehan WM, Mannelli M, Friberg P, Grebe SK, Timmers HJ, et al. Catecholamine metabolomic and secretory phenotypes in phaeochromocytoma. Endocrine-Related Cancer 201118 97-111. (https://doi.org/10.1677/ERC-10-0211)

24 Wu LM, Wu R, Ou YR, Chen BH, Yao QY, Lu Q, Hu J, Jiang M, An DA $\& \mathrm{Xu}$ JR. Fibrosis quantification in hypertensive heart disease with LVH and non-LVH: findings from T1-mapping and contrast-free cardiac diffusion-weighted imaging. Scientific Reports 20177559 (https://doi.org/10.1038/s41598-017-00627-5)

25 Szakács CJ \& Mehlman B. Pathologic changes induced by 1-norepinephrine. American Journal of Cardiology 19605 619-627. (https://doi.org/10.1016/0002-9149(60)90127-2)

26 Van Vliet PD, Burchell HB \& Titus JL. Focal myocarditis associated with pheochromocytoma. New England Journal of Medicine 1966274 1102-1108. (https://doi.org/10.1056/ NEJM196605192742002)

27 Kawel-Boehm N, Maceira A, Valsangiacomo-Buechel ER, VogelClaussen J, Turkbey EB, Williams R, Plein S, Tee M, Eng J \& Bluemke DA. Normal values for cardiovascular magnetic resonance in adults and children. Journal of Cardiovascular Magnetic Resonance 201517 29. (https://doi.org/10.1186/s12968-015-0111-7)

28 Vo HQ, Marwick TH \& Negishi K. MRI-derived myocardial strain measures in normal subjects. Journal of the American College of Cardiologists: Cardiovascular Imaging 201811 196-205. (https://doi. org/10.1016/j.jcmg.2016.12.025)

29 Taylor RJ, Moody WE, Umar F, Edwards NC, Taylor TJ, Stegemann B, Townend JN, Hor KN, Steeds RP, Mazur W, et al. Myocardial strain measurement with feature-tracking cardiovascular magnetic resonance: normal values. European Heart Journal: Cardiovascular Imaging 201516 871-881. (https://doi.org/10.1093/ehjci/jev006)

30 Prejbisz A, Lenders JWM, Eisenhofer G \& Januszewicz A. Cardiovascular manifestations of phaeochromocytoma. Journal of Hypertension 201129 2049-2060. (https://doi.org/10.1097/ HJH.Ob013e32834a4ce9)

31 Rodrigues JCL, Rohan S, Dastidar AG, Trickey A, Szantho G, Ratcliffe LEK, Burchell AE, Hart EC, Bucciarelli-Ducci C, Hamilton MC, et al. The relationship between left ventricular wall thickness, myocardial shortening, and ejection fraction in hypertensive heart disease: insights from cardiac magnetic resonance imaging. Journal of Clinical Hypertension 201618 1119-1127. (https:// doi.org/10.1111/jch.12849)

32 MacIver DH, Adeniran I \& Zhang H. Left ventricular ejection fraction is determined by both global myocardial strain and wall thickness. International Journal of Cardiology. Heart and Vasculature $2015 \mathbf{7}$ 113-118. (https://doi.org/10.1016/j.ijcha.2015.03.007)

33 Wittstein IS, Thiemann DR, Lima JAC, Baughman KL, Schulman SP Gerstenblith G, Wu KC, Rade JJ, Bivalacqua TJ \& Champion HC. Neurohumoral features of myocardial stunning due to sudden emotional stress. New England Journal of Medicine 2005352 539-548. (https://doi.org/10.1056/NEJMoa043046)

34 Mori H, Ishikawa S, Kojima S, Hayashi J, Watanabe Y, Hoffman JI \& Okino H. Increased responsiveness of left ventricular apical myocardium to adrenergic stimuli. Cardiovascular Research 199327 192-198. (https://doi.org/10.1093/cvr/27.2.192)

Received in final form 8 March 2019

Accepted 25 March 2019

Accepted Preprint published online 25 March 2019 https://ec.bioscientifica.com https://doi.org/10.1530/EC-18-0553
(C) 2019 The authors Published by Bioscientifica Ltd

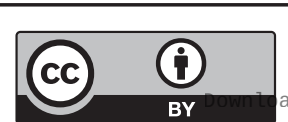

This work is licensed under a Creative Commons Attribution 4.0 International License. ded from Bioscientifica.com at 04/26/2023 07:41:46AM 